\title{
Quejas somáticas atribuidas a factores ambientales: ¿es la sensibilidad química múltiple un nuevo síndrome?
}

\author{
W. Winters, S. Devriese \& O. Van den Bergh \\ Universidad Católica de Lovaina \\ Lovaina, Bélgica
}

Muchos síndromes se caracterizan por tener una gran variedad de manifestaciones en diferentes sistemas orgánicos sin una explicación somática. Tal es el caso de la sensibilidad química múltiple, 'multiple chemical sensitivity' (MCS). Lo más característico de la MCS es que el paciente atribuye sus síntomas a factores del medio ambiente. El MCS se desarolla en dos fases: primero los síntomas se producen como una reacción a una exposición a sustancias químicas; en una etapa posterior, los síntomas aparecen como reacción a otras sustancias que no están relacionadas con la primera exposición. No hay factores explicativos fisiológicos. La teoría psicológica del aprendizaje por condicionamiento pavloviano ofrece evidencia clínica, experimental y también un tratamiento adecuado.

olores / quejas de salud / MCS / condicionamiento / atribución

\section{Somatic complaints attributed to environmental factors: is multiple chemical sensitivity a new syndrome?}

A lot of syndromes are characterized by a great variety of complaints in different organ systems without a somatic explanation. Multiple chemical sensitivity (MCS) is such a syndrome. The most typical characteristic of MCS is the attribution of symptoms to factors in the environment. MCS develops in 2 stages: first, symptoms occur in reaction to a chemical exposure, in a later stage they occur in reaction to other substances that are unrelated to the first exposure. There are no satisfactory physiological explanations. The psychological theory of learning: Pavlovian conditioning offers clinical and experimental evidence and an adequate treatment.

odors / health complaints / MCS / conditioning / attribution

Traducción del inglés por W. Winters.

Dirección del autor: Omer.vandenbergh@psy.kuleuven.ac.be 


\section{INTRODUCCIÓN}

En la literatura científica médica son cada vez más las publicaciones acerca de la MCS. El primer caso fue referido hace 40 años por Theron Randolph, alergista de Chicago (Randolph, 1962). El paciente se enfermaba cada vez que iba a su trabajo y transitaba por una zona industrializada. Casos parecidos se presentaron posteriormente. Desde entonces la cantidad de pacientes con MCS, sobre todo en Estados Unidos, se ha incrementado mucho.

La MCS se relaciona con una variedad de síntomas que se desarrollan en presencia de sustancias químicas y, muchas veces, olorosas. Hasta ahora solamente se ha podido diagnosticar MCS mediante los síntomas subjetivos y no hay una explicación generalmente aceptada. Por eso, el síndrome es controvertido y es tema de muchos debates en congresos, reuniones, revistas médicas y psicológicas (Fiedler \& Kipen, 1997; Ford, 1997; Ziem \& Davidoff, 1992). Muchos especialistas participan en la discusión: toxicólogos, inmunólogos, epidemiólogos, psiquiatras, psicólogos, médicos que trabajan en empresas, y "ecologistas clínicos". En este artículo revisaremos algunos hechos e hipótesis más representativos.

\section{Paciente}

El paciente M. (27 años) se queja de hipersensibilidad a sustancias muy diferentes. Después de un contacto con las sustancias químicas se producen una variedad de síntomas: aturdimiento, dificultades de concentración, sensación de vértigo, dolores de cabeza, ahogo, congestión de pecho y una sensación general de debilidad. Los síntomas no siempre aparecen simultáneamente y varía también la intensidad. Muchas veces duran hasta el día siguiente. Algunos de los materiales causantes de tales malestares son algunos productos de limpieza, pintura, gases de escape, lociones para después de afeitarse, humo de cigarrillos. El humo de combustión provoca las peores quejas. El paciente muestra un comportamiento de evitación: sale de la casa cuando la están limpiando; cuando está conduciendo el auto evita pasar cerca de las fábricas que emanan gases u olores, cierra las ventanas y la ventilación del vehículo. Si siente que el aire está contaminado por la combustión o hay una nube de humo visible se detiene o cambia de ruta.

El problema con este paciente empezó medio año atrás. Estaba trabajando en una industria de material de aislamiento y tenía que manipular productos químicos "peligrosos". Progresivamente aparecieron malestares que fueron empeorando. Mientras tanto mostró un comportamiento de evitación, por ejemplo, manejó los productos muy lenta y cuidadosamente. Muchas veces se quedó enfermo en su casa. Era el único de 10 obreros en la misma situación que tenía los síntomas. Fue 
despedido y, desde entonces, ha quedado desempleado.

\section{Características de los Síntomas}

La gran variedad de síntomas en diferentes sistemas orgánicos es típico en los pacientes de MCS (Miller, 1994). Los más frecuentes son los neuropsicológicos (náuseas, confusión, pérdida de memoria, inestabilidad emocional, cambios de personalidad), constitucional (fatiga, debilidad, pérdida de apetito) y cardiorrespiratorio (disnea, tos, aceleración del ritmo cardiaco, dolor de pecho) (Simon, 1992). Otras quejas son dolores de cabeza, percepción muy fuerte de olores, disfunción cognitiva, sensación de hormigueo y muchas otras no muy específicas (Bronstein, 1997).

Cullen presenta la siguiente descripción:

MCS es un desorden adquirido y caracterizado por síntomas recurrentes, referido a sistemas orgánicos múltiples, que ocurre como respuesta a la exposición demostrable a muchos compuestos químicos que no se relacionan entre sí, en dosis muy por debajo de aquellas establecidas como causantes de efectos dañinos en la población general. Ninguno de los tests de función fisiológica generalmente aceptados demuestra correlación alguna con los síntomas mencionados (Cullen, 1987).

Como se trata de síntomas no muy específicos y subjetivos, hay una gran semejanza con otros diagnósticos (Ford, 1997). Hay síntomas parecidos con la fibromialgia (Ford, 1997), el síndrome de la fatiga crónica (Ford, 1997), el síndrome del edificio enfermo (Redlich, Sparer \& Cullen, 1997), desorden de pánico (Shustermans \& Dager, 1991), el síndrome atípico del estrés postraumático (Schottenfield \& Cullen, 1986), la hiperventilación (Lehrer, 1997) y el asma (Ross, 1997). Sin embargo, lo típico en MCS, es la atribución de síntomas a sustancias ambientales (Sparks et al., 1994).

\section{Desarrollo}

Basándose en observaciones clínicas se sabe que la MCS se desarrolla en dos fases. En la primera se producen los síntomas como consecuencia de la exposición a una sustancia química. Puede ser exposición repentina a grandes cantidades de la sustancia (un suceso accidental por un derrame de gas) (Davidoff, Keyl \& Meggs, 1998), o una exposición continua a pequeñas cantidades (Montgomery \& Reasor, 1994). A veces el estrés extremo, sin exposición química, es responsable del desarrollo de síntomas (Miller, 1994). En la segunda fase, los síntomas aparecen frente a sustancias químicas que se usan a diario, que normalmente son toleradas por la mayoría de la población (humo de cigarro, perfume, gases de los automóviles). Al cabo de algún tiempo más sustancias no relacionadas con la primera exposición provocan también síntomas (alcohol, cafeína, alimentos) (Miller, 1994). 


\section{Prevalencia}

Es difícil ofrecer una estimación confiable acerca de la frecuencia de la MCS. Esto se debe, en parte, al desconocimiento del síndrome y al deficiente reconocimiento de médicos y pacientes. Mucho depende también de la atención que los medios de comunicación prestan al medio ambiente y al tipo de síntomas que se relacionan. Sin embargo, hay estudios que refieren estimaciones de 3 a 5\% en Estados Unidos (Sorg \& Prasad, 1997). Pero es sorprendente que MCS aparece más en algunos lugares que en otros. El rol de las ideas culturales en la atribución de la causa de una enfermedad es muy importante (Davey, 1995; 1997; Williams \& Lees-Haley, 1997; Dewaraj \& Sasaki, 1991). En los países escandinavos, por ejemplo, la incidencia de hipersensibilidad a la electricidad es más frecuente (Arnetz, 1997).

Las diferencias individuales también son importantes: en un grupo que está expuesto a la misma cantidad de sustancias químicas no todos desarrollan los síntomas (Pennebaker, 1994). Estas diferencias tienen que ver con herencia, procesos hormonales, costumbres de la alimentación, factores ambientales o estrés, pero también con diferencias en procesos psicológicos (Miller, 1994; Siegel \& Kreutzer, 1997; Cohen, Kehrl \& Berglund, 1997). Más mujeres que hombres sufren de MCS (Fiedler \& Kipen, 1997; Cullen, 1987). Pennebaker (1994) sostiene que esto se debe a las diferencias que hay entre uno y otro sexo en cuanto al reporte de los síntomas, y también en relación con otros aspectos, como por ejemplo la percepción de olores.

\section{EXPLICACIONES}

La controversia principal sobre la MCS radica en la explicación -psicológica o fisiológica- de los síntomas (Ziem \& Davidoff, 1992). Esto implica generalmente una visión dual sobre las enfermedades, ya sea responsable el cuerpo o la mente. Nuestra opinión es que los factores psicológicos y fisiológicos no son incompatibles y que para cada uno de los factores se debe encontrar evidencia positiva. Para decir que es "psicológico" igual que para decir que es "fisiológico" hay que identificar un proceso explicativo responsable en lugar de decir "si no es fisiológico entonces, tiene que ser psicológico" sin relacionar esto con un proceso. Hasta ahora no hay factores fisiológicos suficientemente satisfactorios. Las teorías psicológicas generalmente son identificadas, demasiado, con "imaginación” y "sugestión". Eso implica que hay que convencer a los pacientes para que se den cuenta de sus ideas falsas. Esta opinión implica que todos los procesos psicológicos se desarrollan conscientemente y que es posible controlarlos voluntariamente. Esto no pasa por los procesos mentales que intervienen en el condicionamiento clásico, propuesto por varios autores como el mecanismo principal en 
MCS (Simon, 1992; Schottenfield \& Cullen, 1986; Sparks et al., 1994; Siegel \& Kreutzer, 1997; Shusterman, 1992; Bolla-Wilson et al., 1988; MacPhail, 1997). Esta teoría ya ha probado su efectividad en la explicación y tratamiento de las fobias (Davey, 1997).

\section{CONDicionamiento CLÁSICO:}

\section{LA TEORÍA}

El condicionamiento clásico es un mecanismo de la teoría del aprendizaje, que va asociado al nombre de Pavlov, por lo que se suele hablar de condicionamiento pavloviano. A pesar de que su principio es extremadamente sencillo y conocido por todos, vamos a recordarlo brevemente. Pavlov realizó sus célebres experimentos con perros. Cuando se le presenta un plato de comida, el perro saliva. Al principio, Pavlov le hacía oír al perro el sonido de un metrónomo antes de darle la comida. Después, una vez que hubo repetido sistemáticamente esta asociación, sometió el perro al ruido del metrónomo pero suprimió la comida, a pesar de lo cual, el perro volvió a salivar. Pavlov dedujo que el plato de comida constituía el estímulo obligatorio y, el metrónomo, el estímulo condicionante. A partir de ese resultado, Pavlov elaboró una teoría del aprendizaje que explicaba por este tipo de condicionamiento todos los comportamientos observables en el ser humano y en los animales.

En la aplicación del condicionamiento clásico al MCS, el estímulo incondicionado (EI) es una exposición tóxica que provoca automáticamente una reacción: la respuesta incondicionada (RI). Un estímulo neutro que ocurre simultáneamente con el EI, por ejemplo un olor, puede volverse un estímulo condicionado (EC) cuando este estímulo provoca por sí mismo una reacción muy parecida a la RI. Esta reacción se llama la respuesta condicionada (RC).

EC

EI

Respuesta (RI / RC)

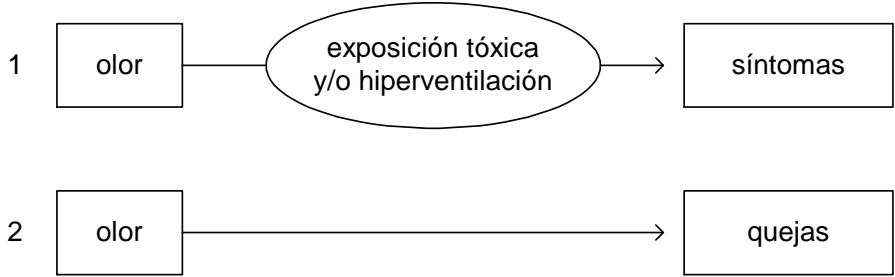

$\mathrm{EC}=$ estímulo condicionado

$\mathrm{EI}=$ estímulo incondicionado

$\mathrm{RI}=$ respuesta incondicionada

$\mathrm{RC}=$ respuesta condicionada

MCS $=$ Multiple Chemical Sensitivity

$1=$ primera fase

2 = segunda fase 
Cabe destacar en la teoría del aprendizaje los mecanismos de "generalización” y "extinción”. La generalización ocurre cuando la reacción condicionante (quejas aprendidas, provocadas por un olor) se generaliza a otros olores, no relacionados con la exposición tóxica principal. La extinción se refiere al fenómeno de la reacción aprendida que extingue en ciertas condiciones después de exposiciones repetitivas al EC.

Cada vez que se confronta con las quejas corporales sin que exista un proceso somático, se podría investigar si el condicionamiento clásico podría explicarlas. Esto también es posible con síndromes conocidos, tales como el síndrome de la hiperventilación, en que se observa sistemáticamente las quejas típicas sin baja de $\mathrm{CO}_{2}$ (Hornsveld, Garssen, Dop \& Van Spiegel, 1990). Aquí el condicionamiento clásico como explicación es muy probable y evita que el HVS sea desestimado como tal (Stegen, Van den Bergh \& Van den Woestijne, 1996).

\section{CONDICIONAMIENTO CláSICO:}

\section{EVIDENCIA CLÍNICA Y EXPERIMENTAL}

En la literatura se refieren muchos casos cuyos síntomas se desarollan según este esquema (Dager et al., 1987; Shusterman, Balmes \& Cones, 1988). Casi nunca se refiere a quejas de MCS con sustancias sin olores como plomo y arsénico (Bolla-Wilson et al., 1988).

Probamos la explicación en algunos estudios usando un paradigma de con- dicionamiento respiratorio diferencial con olores como estímulos condicionantes y $7,5 \%$ de inhalaciones de $\mathrm{CO}_{2}$ como estímulo incondicionante (Van den Bergh et al., 1995; 1997; 1998; 1999; Stegen et al., 1999). Fue hallado un efecto de condicionamiento: una exposición de baja toxicidad provocó quejas de salud hacia olores que por sí no podrían explicar las quejas. No solamente fue hallado un efecto con olores (solamente los negativos) pero también con pensamientos (Stegen et al., 1999). El primer mecanismo podría intervenir en MCS, el último en HVS.

\section{IMPLICACIONES TERAPÉUTICAS}

Fuera de la evidencia teórica, clínica y experimental, la teoría del condicionamiento pavloviano tiene la ventaja de poder ofrecer un tratamiento adecuado. Las reacciones aprendidas pueden ser cambiadas por métodos de esta teoría misma (extinción, contracondicionamiento, revaluación). En otros estudios se ha hallado que las quejas aprendidas hacia los olores pueden ser extinguidas, desapareciendo al ser expuestas varias veces sólo al EC (Van den Bergh et al., 1999).

Un tratamiento terapéutico conductual típico considerará los siguientes métodos: a) exposición larga al EC sin admitir comportamiento de evitación; b) enseñar otras reacciones al EC, que son incompatibles con la RC (como relajamiento); y, c) cambiar las experiencias e ideas de manera cognitiva. Si la 
hiperventilación es importante, también tiene que ser tratada en fase 1 o 2 pero no fuera de esas fases, como tratamiento en sí de la respiración. El efecto de contracondicionamiento ya ha sido hallado en algunos estudios clínicos de Bolla-Wilson et al. (1988).

\section{Tratamiento del PaCiente M}

Después de ser referido por el médico al especialista, todas las evidencias de malestares somáticos fueron negativas. En observaciones más detalladas, se halló que sustancias olorosas provocaron inspiraciones hondas y movimientos incontrolados de respiración y deglución como si se quisiera, de este modo, expulsar las sustancias químicas de su cuerpo. La consecuencia fue un estado de aerofagia. El conjunto de quejas se asemejaba a una reacción de hiperventilación (HV) condicionada hacia olores. La aerofagia provocó eructos del aire tragado, así que los olores volvieron y la reacción de HV fue provocada de nuevo. Cada exposición con consecuencias negativas confirmó la ansiedad anticipatoria que estimuló una siguiente reacción. El tratamiento fue una terapia de exposición. En primer lugar se enseñó una reacción relajante y un control adecuado de la respiración. Después hubo que aplicarlas durante exposiciones graduales a las sustancias críticas. Al final recibió tareas para la casa: tenía que buscar sustancias y situaciones olorosas y aplicar las habilidades aprendidas en la reali- dad. Después de ocho sesiones ya no tenía síntomas.

\section{ConClusión}

Todavía queda mucho por explorar en este campo de la MCS. Para el paciente es importante que su enfermedad sea primero reconocida, no sólo para un tratamiento adecuado sino también para no ser referido a instancias jurídicas o médicas con todas las consecuencias psicológicas. Para empleadores y empresas es importante saber su rol y su responsabilidad relativos a los pacientes. Por otro lado, es también importante que no se diagnostique MCS en cualquier situación, porque esta "enfermedad de la civilización" es creada en parte por los médicos, la ciencia y los medios de comunicación. Un buen diagnóstico, hecho por un grupo multidisciplinario (toxicólogos, psicólogos), es una condición importante. 


\section{REFERENCIAS}

Arnetz, B.B. (1997). Technological stress: psychophysiological aspects of working with modern information technology. Scandinavian Journal of Work and Environmental Health, 23 (suppl. 3), 97-103.

Bolla-Wilson, K., Wilson, R. J., \& Bleeker, M.L. (1988). Conditioning of physical symptoms after neurotoxic exposure. Journal of Occupational Medecine, 30, 684-686.

Bronstein, A.C. (1995). Multiple chemical sensitivities - New paradigm needed. Clinical Toxicology, 33, 93-94.

Cohen, N., Kehrl, H., et al. (1997). Psychoneuroimmunology. Environmental Health Perspectives, 105 (suppl. 2), 527-530.

Cullen, M.R. (1987). The worker with multiple chemical hypersensitivities: An overview. Occupational Medicine, 2, 655-661.

Dager, S.R., Holland, J.P., Cowley, D.S., \& Dunner, D.L. (1987). Panic disorder precipitated by exposure to organic solvents in the workplace. American Journal of Psychiatry, 144, 1056-1058.

Davey, G. (1995). Preparedness and phobias: specific evolved associations or a generalized expectancybias. Behavioral and Brain Sciences, 18, 289-325.

Davey, G.C.L. (1997). A conditioning model of phobias. Phobias: A handbook of theory, research and treatment (pp. 301-322).

Davidoff, A.L., Keyl, P.M., \& Meggs, W. (1998). Development of multiple chemical sensitivities in laborers after acute gasoline fume exposure in an un- derground tunneling operation. Archives of Environmental Health, 53(3), 183-189.

Dewaraja, R., \& Sasaki, Y. (1991). Semenloss syndrome: A comparison between Sri Lanka and Japan. American Journal of Psychotherapy, 45(1), 14-20.

Fiedler, N., \& Kipen, H. (1997). Chemical sensitivity: The scientific literature. Environmental Health Perspectives, 105 (suppl. 2), 409-416.

Ford, C.V. (1997). Somatization and fashionable diagnoses: illness as a way of life. Scandinavian Journal of Work and Environmental Health, 23 (suppl.3), 716.

Hornsfeld, H., Garssen, B., Dop, M., \& Van-Spiegel, P. (1990). Symptom reporting during voluntary hyperventilation and mental load: Implications for diagnosing Hyperventilation Syndrome. Journal of Psychosomatic Research, 34(6), 687-697.

Lehrer, P.M. (1997). Psychophysiological hypotheses regarding multiple chemical sensitivity syndrome. Environmental Health Perspectives, 105 (suppl. 2), 479-484.

MacPhail, R.C. (1997). Evolving concepts of chemical sensitivity. Environmental Health Perspectives, 105 (suppl. 2), 455-456.

Miller, C.S. (1994). Chemical sensitivity: History and phenomenology. Toxicology and Industrial Health, 10(4/5), 253276.

Montgomery, M.R., \& Reasor, M.J. (1994). A toxicologic approach for evaluating cases of sick building syndrome or multiple chemical sensitivity. Journal of 
Allergy and Clinical Immunology, 94(2), 371-375.

Pennebaker, J.W. (1994). Psychological bases of symptom reporting: perceptual and emotional aspects of chemical sensitivity. Toxicology and Industrial Health, 10(4,5), 497-509.

Randolph, T.G. (1962). Human ecology and susceptibility to the chemical environment. Springfield, IL: Charles C. Thomas.

Redlich, C.A., Sparer, J., \& Cullen, M.R. (1997). Sick building syndrome. The Lancet, 349, 1013-1016.

Ross, G.H. (1997). Clinical characteristics of chemical sensitivity: An illustrative case history of asthma and MCS. Environmental Health Perspectives, 105 (suppl. 2), 437-442.

Schottenfeld, R.S., \& Cullen, M.R. (1986). Recognition of occupation-induced posttraumatic stress disorders. Journal of Occupational Medecine, 28(5), 365369.

Shusterman, D.J. (1992). Critical review: The health significance of environmental odor pollution. Archives of Environmental Health, 47, 76-87.

Shusterman, D.J., \& Dager, S.R. (1991). Prevention of psychological disability after occupational respiratory exposures. Journal of Occupational Medecine, 6, 11-27.

Shusterman, D., Balmes, J., \& Cones, J. (1988). Behavioral sensitization to irritants/odorants after acute overexposures. Journal of Occupational Medecine, 30(7), 565-567.

Siegel, S., \& Kreutzer, R. (1997). Pavlovian conditioning and multiple chemi- cal sensitivity. Environmental Health Perspectives, 105 (suppl. 2), 521-526.

Simon, E.G. (1992). Psychiatric treatments in multiple chemical sensitivity. Toxicology and Industrial Health, 8(4), 6772 .

Sorg, B.A., \& Prasad, B.M. (1997). Potential role of stress and sensitisation in the development and expression of multiple chemical sensitivity. Environmental Health Perspectives, 105 (suppl. 2), 467-472.

Sparks, P.J., Daniel, W., Black, D.W., Kipen, H.M., Altman, L.C., Simon, G.E., \& Terr, A.I. (1994). Multiple chemical sensitivity syndrome: A clinical perspective. Journal of Occupational Medecine, 36, 718-730.

Stegen, K., De Bruyne, K., Rasschaert, W., Van de Woestijne, K.P., \& Van den Bergh, O. (1999). Fear-relevant images as conditioned stimuli for somatic complaints, respiratory behavior and reduced end-tidal $\mathrm{pCO}_{2}$. Journal of $\mathrm{Ab}$ normal Psychology, 108, 143-152.

Stegen, K., Van den Bergh, O., \& Van de Woestijne, K.P. (1996). Psychosomatische klachten kun je leren: Conditionering van psychosomatische klachten via toediening van $\mathrm{CO}_{2}$-rijke lucht. Gedrag \& Gezondheid, 24(4), 233-240.

Van den Bergh, O., Kempynck, P.J., Van de Woestijne, K.P., Baeyens, F., \& Eelen, P. (1995). Respiratory learning and somatic complaints: A conditioning approach using $\mathrm{CO}_{2}$-enriched air inhalation. Behavior Research and Therapy, 33(5), 517-527.

Van den Bergh, O., Stegen, K., \& Van de Woestijne, K.P. (1997). Learning to have psychosomatic complaints: Con- 
ditioning of respiratory behavior and somatic complaints in psychosomatic patients. Psychosomatic Medecine, 59, 13-23.

Van den Bergh, O., Stegen, K., \& Van de Woestijne, K.P. (1998). Memory effects on symptom reporting in a respiratory learning paradigm. Health Psychology, 17(3), 241-248.

Van den Bergh, O., Stegen, K., et al. (1999). Acquisition and extinction of somatic complaints in response to odors: a new paradigm to investigate multiple chemical sensitivity. Oсcupational and Environmental Medicine, 56, 295-301.

Williams, C.W., \& Lees-Haley, P.R. (1997). Effect of information about odor on causal ascriptions for illness. Perceptual and Motor Skills, 85(2), 411-418.

Ziem, G.E., \& Davidoff, L.L. (1992). Illness from chemical odors: Is the health significance understood? Archives of Environmental Health, 47, 88-91. 